\title{
17 $\beta$-Estradiol Differentially Regulates Stress Circuitry Activity in Healthy and Depressed Women
}

\author{
Emily G Jacobs*, 1,2,3, Laura M Holsen ${ }^{1,2,3}$, Katie Lancaster ${ }^{4}$, Nikos Makris ${ }^{3,5}$, Sue Whitfield-Gabrieli ${ }^{5,6}$, \\ Anne Remington ${ }^{1,2}$, Blair Weiss ${ }^{2}$, Stephen Buka ${ }^{7}$, Anne Klibanski ${ }^{8}$ and Jill M Goldstein ${ }^{1,2,3,5}$ \\ 'Division of Women's Health, Department of Medicine, Brigham and Women's Hospital, Boston, MA, USA; '2Department of Psychiatry, Brigham \\ and Women's Hospital, Boston, MA, USA; ${ }^{3}$ Harvard Medical School, Boston, MA, USA; ${ }^{4}$ Department of Psychology, University of Virginia, \\ Charlottesville, VA, USA; ${ }^{5}$ Athinoula A. Martinos Center, Massachusetts General Hospital and Massachusetts Institute of Technology, \\ Charlestown, MA, USA; ${ }^{6}$ Department of Brain and Cognitive Sciences, Massachusetts Institute of Technology, Cambridge, MA, USA; ${ }^{7}$ Department \\ of Community Health, Brown University, Providence, RI, USA; ${ }^{8}$ Neuroendocrine Unit, Department of Medicine, Massachusetts General Hospital, \\ Boston, MA, USA
}

Many regions within stress neurocircuitry, including the anterior hypothalamus, amygdala, hippocampus, and medial prefrontal cortex, are densely populated with sex steroid receptors. Substantial evidence from animal studies indicates that the gonadal hormone $17 \beta$-estradiol $\left(E_{2}\right)$ impacts the structure and function of these regions, but human studies are limited. Characterizing estradiol's role in stress circuitry in vivo in humans may have important clinical implications given the comorbidity between major depressive disorder (MDD), stress circuitry dysfunction and endocrine dysregulation. In this study, we determined estradiol's role in modulating activity within cortical and subcortical stress circuitry regions in healthy and MDD women. Subjects were part of a population-based birth cohort, the New England Family Study. Capitalizing on the endogenous fluctuation in $E_{2}$ during the menstrual cycle, we conducted a within-person repeatedmeasures functional neuroimaging study in which 15 women with recurrent MDD, in remission, and 15 healthy control women underwent hormonal evaluations, behavioral testing, and $\mathrm{fMRI}$ scanning on two occasions, under low and high $\mathrm{E}_{2}$ conditions. Subjects completed an $\mathrm{AMRI}$ scan while undergoing a mild visual stress challenge that reliably activated stress neural circuitry. Results demonstrate that $E_{2}$ modulates activity across key stress circuitry regions, including bilateral amygdala, hippocampus, and hypothalamus. In healthy women, robust task-evoked BOLD signal changes observed under low $E_{2}$ conditions were attenuated under high $E_{2}$ conditions. This hormonal capacity to regulate activity in stress circuitry was not observed in MDD women, despite their remitted status, suggesting that dysregulation of gonadal hormone function may be a characteristic trait of the disease. These findings serve to deepen our understanding of estradiol's actions in the healthy brain and the neurobiological mechanisms that may underlie the pronounced sex difference in MDD risk.

Neuropsychopharmacology (20I5) 40, 566-576; doi:I0.1038/npp.2014.203; published online 17 September 20I4

\section{INTRODUCTION}

The stress response is a highly conserved, tightly coordinated neuroendocrine and autonomic nervous system response that equips an organism with the ability to achieve homeostasis following a stressor. Persistent maladaptive or hyperactive responses can lead to mood and anxiety disorders, including major depressive disorder (MDD). The neural circuitry involved in the appraisal of potential stressors and the regulation of hypothalamic-pituitaryadrenal (HPA) axis output includes medial prefrontal cortex (mPFC), hippocampus, amygdala, and anterior hypothalamus (Ulrich-Lai and Herman, 2009). These regions provide

*Correspondence: Dr EG Jacobs, Harvard Medical School, Brigham and Women's Hospital, One Brigham Circle, Boston, MA 02120, USA, Tel: + | 617525 8637, Fax: + | 617525 7900;

E-mail: egjacobs@nmr.mgh.harvard.edu

Received 28 April 2014; revised I August 2014; accepted 2 August 2014; accepted article preview online 12 August 2014 regulatory feedback to the HPA axis and are themselves major targets of adrenal stress hormones. A distinct characteristic of these regions is that they express glucocorticoid and sex steroid receptors (MacLusky et al, 1987; Clark et al, 1988; Handa et al, 1994; Kawata, 1995; Tobet and Hanna, 1997; Donahue et al, 2000; Östlund et al, 2003). In fact, many of the brain regions that regulate the release of adrenal steroid hormones, including the hypothalamus, amygdala, hippocampus, and mPFC, are densely populated with estrogen receptors (ERs) $\alpha$ or $\beta$ (Österlund et al, 2000; Österlund and Hurd, 2001; Perlman et al, 2005; Wang et al, 2010). Interactions between the HPA and HP-gonadal (HPG) axes are well known. For example, glucocorticoids can inhibit reproductive function (Nikolarakis et al, 1986; Wingfield and Sapolsky, 2003) and, reciprocally, HPG axis hormones can act at the level of the hypothalamus to control the release of adrenal stress hormones (Weiser et al, 2008). Preclinical evidence suggests that $17 \beta$-estradiol also acts 'upstream' of the hypothalamus to regulate 
the structure and function of a distributed set of brain regions within stress neural circuitry (Nabekura et al, 1986; Wooley, 1998; Hao et al, 2006; Walf and Frye, 2006; Weiser et al, 2008).

A long history of clinical evidence implicates HPA- and HPG-axis dysregulation in the development of mood disorders (Plotsky et al, 1998; Young and Korszun, 2002; Nemeroff et al, 1984). Throughout the lifecourse, changes in women's reproductive status have been associated with increased risk for mood disturbance and MDD (Rabin et al, 1990; Baischer et al, 1995; Rubinow and Schmidt, 1996, 2006; Harlow et al, 2003; Payne, 2003; Roca et al, 2003; Spinelli, 2005; Payne et al, 2009; Freeman et al, 2014). MDD incidence increases with pubertal onset in females (Angold and Costello, 2006), chronic use of oral contraceptives (Young et al, 2007), the postpartum period (Bloch et al, 2000; Brummelte and Galea, 2010), and perimenopause (Schmidt and Rubinow, 2009). A recent longitudinal population-based study of women found that the risk of depressive symptoms dropped significantly following menopause. Additional analysis of HPG axis hormones revealed that women with a faster rate of change in follicle-stimulating hormone (FSH) levels (suggesting a faster transition to menopause) had the lowest risk (Freeman et al, 2014). Further, some randomized control trials have found antidepressant effects of shortterm estradiol administration in perimenopausal women (Schmidt et al, 2000; Soares et al, 2001; but see Morrison et al, 2004), which parallels animal studies demonstrating potent anxiolytic effects of $\operatorname{ER} \beta$ agonists in ovariectomized female rodents (Weiser et al, 2010, Oyola et al, 2012). Further, population studies suggest that ovarian dysfunction precedes MDD onset (Nemeroff et al, 1984; Harlow et al, 2003). Some reports have found that MDD patients in an episode have abnormal estradiol (Young et al, 2000) and FSH levels (Daly et al, 2003; but see Saletu et al, 1996; Schmidt et al, 2002).

In a previous in vivo brain-imaging study of healthy women, we found that the activation of stress circuitry regions in response to a mild stress challenge was modulated across the menstrual cycle, strongly implicating sex steroids in the regulation of this circuitry (Goldstein et al, $2005,2010)$. In the current study, we used a within-sample repeated-measures functional MRI (fMRI) design in a new sample of healthy and remitted MDD women to directly test whether $17 \beta$-estradiol modulates activity within stress neural circuitry in healthy women. Further, we examined whether the hormonal regulation of these regions is disrupted in fully or partially remitted MDD women and thus may represent a characteristic trait of the diesease. Taken together, these findings serve to deepen our understanding of estradiol's actions in the healthy brain and the neurobiological mechanisms underlying the pronounced sex difference in MDD risk.

\section{MATERIALS AND METHODS}

\section{Overview}

In a repeated-measures within-person design, 15 healthy adult women and 15 women with remitted major depression (MDD) underwent hormonal evaluations, clinical and cognitive assesments, and fMRI scanning on two occasions, once during the estimated early follicular menstrual cycle phase and once during the estimated late follicular/midcycle phase, order counter-balanced. Subjects underwent fMRI scanning during a mild visual stress challenge. Neutral valence/ low arousal and negative valence/high arousal images were selected from the International Affective Picture System (IAPS) (CSEA-NIMH, 1999), which are known to reliably invoke stress circuitry (Goldstein et al, 2005, 2010; Holsen et al, 2011, 2013). Previous studies have demonstrated a lack of habituation in arousal responses to IAPS images, validating the use of these images at two points in time (Bradley et al, 1996) including with this fMRI task (Goldstein et al, 2005). Morning fasting serum was collected to assay HPG axis hormones. Mood states were evaluated before and after scanning to assess state- and trait-level anxiety and mood.

\section{Subjects}

Participants were selected from the 17741 Boston and Providence pregnancies from the Collaborative Perinatal Project (CPP), known as the New England Family Study (NEFS) (Goldstein et al, 2010). The CPP was a prospective study initiated over 50 years ago to investigate the prenatal and familial antecedents of pediatric, neurological, and psychological disorders of childhood (Niswander and Gordon, 1972). Women (recruited in 1959-1966) were representative of patients receiving prenatal care, thus unselected for psychiatric status. Across NIMH-funded adult follow-up studies of the offspring, we identified 1217 subjects, 511 diagnosed with DSM-IV MDD and 706 healthy controls (HCs) (ORWH-NIMH P50 SCOR MH082679). Systematic structured clinical interviews on all potential subjects were conducted by trained MA-level clinical interviewers. Expert diagnosticians reviewed all information to determine final best estimate diagnoses. Of the 511 MDD cases, 207 had recurrent episodes, of which 148 were women. Cases thus represent a general population sample of adults with MDD, as it is expressed in the community.

Among those subjects re-recruited for brain imaging, we present here 15 recurrent MDD women, in remission, and 15 healthy control women (HC) who were comparable on ethnicity (all Caucasian), right-handedness, socioeconomic status, and general intelligence and were not on a hormonal contraceptive or other HPG axis hormone-based medication. (Table 1). Selection of this subsample for brain imaging was based on the current mood status and MRI exclusion criteria. Given the hypotheses of the current study regarding gonadal hormone regulation of stress circuitry, two controls and four cases were excluded, because serum estradiol and FSH levels indicated that the women were postmenopausal at the time of scanning. None of the women included in analyses were postmenopausal based on menstrual cycle histories (all regularly cycling) and scanday pituitary and gonadal hormone profiles (ranges: LH, 1$40 \mathrm{mIU} / \mathrm{ml}$; FSH, $4-25 \mathrm{mIU} / \mathrm{ml}$ except one; $17 \beta$-estradiol, $17-384 \mathrm{pg} / \mathrm{ml}$; progesterone, $0.01-12.5 \mathrm{ng} / \mathrm{ml}$ ). One MDD woman may have been perimenopausal at the time of her second visit $(\mathrm{FSH}>25 \mathrm{mIU} / \mathrm{ml})$. However, her menstrual cycles were regular, and she exhibited substantial modulation of estradiol between visits $(20.0-115.86 \mathrm{pg} / \mathrm{ml})$ and was therefore not excluded from the analyses. Thus the final sample consisted of 13 healthy controls and $11 \mathrm{MDD}$ 
Table I Demographic and Clinical Characteristics in Healthy and MDD Women

\begin{tabular}{|c|c|c|c|c|c|c|c|}
\hline \multirow[t]{2}{*}{ Characteristic } & \multicolumn{2}{|c|}{$\begin{array}{c}\text { Healthy } \\
\text { controls }(\mathrm{HC})(n=13)\end{array}$} & \multicolumn{2}{|c|}{$\begin{array}{c}\text { Remitted MDD } \\
\text { women (MDD) }(n=I I)\end{array}$} & \multirow[t]{2}{*}{$\begin{array}{l}\text { Between-group } \\
\text { comparisons }\end{array}$} & \multirow[t]{2}{*}{$t$} & \multirow[t]{2}{*}{$P$ value } \\
\hline & Mean & SD & Mean & SD & & & \\
\hline Age (years) & 45.2 & 2.2 & 47.4 & 2.1 & $\mathrm{MDD}>\mathrm{HC}$ & 4.01 & 0.001 \\
\hline BMI & 28.4 & 7.9 & 29.6 & 6.2 & & & \\
\hline Estimated full scale $1 Q^{b}$ & 106.3 & 13.6 & 104.0 & 12.2 & & & \\
\hline Age at symptom onset (years) & - & & 23.3 & 9.0 & & & \\
\hline Duration of illness (years) & - & & 24.3 & 9.5 & & & \\
\hline \multirow[t]{2}{*}{ Duration of recovery (years) } & - & & 5.7 & 6.1 & & & \\
\hline & $n$ & $\%$ & $n$ & $\%$ & & & \\
\hline \multicolumn{8}{|l|}{ Comorbid diagnosis } \\
\hline Current $^{\mathrm{d}}$ & - & - & 4 & 36.3 & & & \\
\hline Past $^{\mathrm{e}}$ & - & - & 4 & 36.3 & & & \\
\hline
\end{tabular}

a Parental socioeconomic status (SES) was a composite index of family income, education, and occupation and ranged from I.7 (low) to 8.3 (high).

${ }^{b}$ Full scale IQ estimated using the sum of age-scaled scores from the WAIS-R Vocabulary and Block Design subtests, and the conversion table C-37 from Sattler, 1992 (p. $85 \mathrm{I})$.

'6 MDD women were currently taking psychotropic medications: sertraline, venlafaxine, fluoxetine $(n=3)$, bupropion, citalopram, methylphenidate.

${ }^{\mathrm{d} C}$ Current comorbid Axis I diagnoses in the MDD group included: three subjects with Panic Disorder, without Agorophobia; one subject with Dysthymic Disorder; and one subject with Specific Phobia, Situational Type. In the HC group, there were no subjects with current Axis I diagnoses.

ePast comorbid Axis I diagnoses in the MDD group included: two subjects with Alcohol Abuse; one subject with Alcohol Dependence; one subject with Cannabis Abuse; and one subject with Cocaine Abuse. In the $\mathrm{HC}$ group, there were no subjects with past Axis I diagnoses.

women, scanned twice, producing a total of 48 data sets. As participants mothers' were recruited during the NEFS study period (1959-1966), women were between ages 43-50 years with MDD women slightly older than HCs. MDD women had generally experienced two or more episodes (two experienced a continuous episode over years rather than discrete multiple episodes). Full remission was defined as not having experienced any MDD symptoms for $\geqslant 30$ days prior to scanning. Two women were in partial remission due to continuing loss of interest and mild guilt but no other symptoms. Healthy controls did not meet DSM-IV criteria for any current Axis 1 disorders. Comorbid current and past Axis 1 diagnoses are reported in Table 1. Healthy controls were not taking any psychotropic medication. No subject was taking a hormone-based medication, including oral contraceptives or hormone replacement therapy.

\section{Study Design and Procedures}

Human subjects' approval was granted by the Partners Healthcare and Brown University. Written informed consent was obtained from all participants. Subjects tracked their menstrual cycles for 3-4 months using a standardized written diary to allow for targeted scheduling based on cycle stage. Participants underwent behavioral testing, hormonal evaluations, and fMRI scanning on two occasions, once during the early follicular phase and once during the late follicular/midcycle phase, order counter-balanced. The two test sessions were categorized as 'low estradiol' or 'high estradiol' based on the relative change in serum estradiol concentrations within a participant. Thus categorization was based on actual steroid hormone concentrations and not solely on menstrual cycle reports. After a morning fasting blood draw (see below), participants were given a small standardized breakfast and administered questionnaires until fMRI scanning commenced. Remitted MDD women completed the 17-item Hamilton Rating Scale for Depression (HAM-D) with trained study staff to assess current mood state. Of the 11 MDD subjects included in the analyses, 2 scored between 8 and 12 during one visit indicating mild depressive symptoms and 9 scored less $<7$ indicating that they were not clinically symptomatic. As most MDD subjects were clinically asymptomatic, findings likely represent MDD 'trait' characteristics rather than moderate or severe 'clinical state'. Mood and anxiety levels were assessed on all subjects using the Profile of Mood States (POMS) and the Spielberger State-Trait Anxiety Inventory (STAI) administered prescanning and postscanning.

\section{Endocrine Measures}

Trained nurses inserted a saline-lock IV line in the nondominant forearm and acquired a fasting (since midnight) morning blood at approximately 0900 hours to evaluate 
HPG axis hormone concentrations, including sex steroids (estradiol, progesterone, and testosterone) and gonadotropins ( $\mathrm{LH}$ and follicle-stimulating hormone (FSH)). HPA axis hormones were also assessed but were not the subject of current analyses. Approximately $30 \mathrm{cc}$ of blood were sampled, allowed to clot for 45-60 min, spun, aliquoted, and stored frozen at $-80^{\circ} \mathrm{C}$. Serum hormones were analyzed in duplicate with commercial immunoassay kits (dynamic range, precision): $17 \beta$-estradiol $(20-4800 \mathrm{pg} / \mathrm{ml}, 12-21 \%)$, progesterone $(0.08-40 \mathrm{ng} / \mathrm{ml}, 6.6-9.5 \%)$, testosterone $(10-$ $1600 \mathrm{ng} / \mathrm{dl}, 1.67-3.93 \%)$, LH $(0.2-250 \mathrm{mIU} / \mathrm{ml}, 4.3-6.4 \%)$, FSH (0.2-200 mIU/ml; 3.1-4.3\%), sex hormone-binding globulin (SHBG) $(0.33-200 \mathrm{nmol} / \mathrm{l}, 4.5-4.8 \%)$ : Immunoradiometric Assay (IRMA), DiaSorin, Inc., Stillwater, MN.

\section{Visual Stress Challenge Task}

Subjects performed a mild visual stress challenge fMRI paradigm in which they viewed negative valence/high arousal and neutral valence/low arousal images adapted from the IAPS, as described and validated previously in multiple studies (Goldstein et al, 2005, 2010; Holsen et al, 2011, 2013) (Figure 1). Fixation stimuli were created using Fourier transforms of neutral valence/low arousal images. Briefly, participants viewed blocks of negative, neutral, and fixation images and were instructed to press a button when a new image appeared in order to ensure attention. The task consisted of three 6-min runs; each run consisted of four blocks each of negative, neutral, and fixation trials, and each 30 -s block consisted of a new image presented every 2 s. For additional experimental details, see Goldstein et al (2005). Following the fMRI session, participants were shown two blocks of negative and neutral images presented on a computer outside of the scanner and provided subjective ratings of arousal using the IAPS Self-Assesment Manikin task.

\section{fMRI Data Acquisition and Analysis}

fMRI scanning was conducted on a Siemens Tim Trio 3T MR scanner with a 12-channel head coil. In all, 180 functional volumes were acquired using a spin echo, $\mathrm{T} 2{ }^{*}$ weighted sequence $(\mathrm{TR}=2000 \mathrm{~ms} ; \mathrm{TE}=40 \mathrm{~ms} ; \mathrm{FOV}=200$ $\times 200 \mathrm{~mm}$; matrix $=64 \times 64$; in-plane resolution $=3.125 \mathrm{~mm}$; slice thickness $=5 \mathrm{~mm} ; 23$ contiguous slices aligned to the
AC-PC plane). fMRI data were preprocessed using Statistical Parametric Mapping (SPM8) (Wellcome Department of Cognitive Neurology, 2008) and custom routines in MATLAB (Mathworks, Inc., 2000) and included realignment and geometric unwarping of EPI images using magnetic fieldmaps, correction for bulk-head motion, nonlinear volume-based spatial normalization (standard Montreal Neurological Institute brain template), spatial smoothing with a Gaussian filter ( $6 \mathrm{~mm}$ at FWHM), and artifact detection (https://www.nitrc.org/projects/artifact_detect) to identify outliers in the global mean image time series (threshold: 3.5) and movement (threshold: 0.7; measured as scan-to-scan movement, separately for translation and rotation) parameters. The number of outliers did not differ significantly between $\mathrm{MDD}(\mathrm{M}=7.25, \mathrm{SD}=6.78)$ and $\mathrm{HC}$ women $(\mathrm{M}=9.50, \mathrm{SD}=13.76, p=0.51)$. Outliers were entered as nuisance regressors in the first-level, singlesubject GLM analysis. Using a block design, each epoch of trials was modeled using a boxcar function convolved with a canonical hemodynamic response function. Specific comparisons of interest (negative vs neutral blood oxygen-level dependent (BOLD) signal changes) from single-subject analysis were then tested using linear contrasts. Contrast images from the individual subject level were submitted to a second-level analysis in which subjects were treated as a random effect.

To perform region of interest (ROI) analyses, a priori ROIs (hypothalamus, amygdala, hippocampus, mPFC) were first anatomically defined using a manually segmented MNI-152 brain (based on methods previously published by the Center for Morphometric Analysis at Massachusetts General Hospital and Harvard Medical School; see Makris et al, 2013) and implemented as overlays on the SPM8 canonical brain using the Wake Forest University PickAtlas ROI toolbox for SPM (Maldjian et al, 2003). Given hypotheses about specific brain regions, we used the small volume correction approach in SPM8, which limits voxelwise analyses to voxels within a priori ROIs. Statistical maps comparing negative $>$ neutral images were generated at voxel-wise family-wise error (FWE)-corrected $p<0.05$ based on 'supergroup' task-evoked activity, independent of estradiol status (see Supplementary Figure S2). These maps represent data from subjects' two test sessions so as not to bias analyses that directly compare low and high

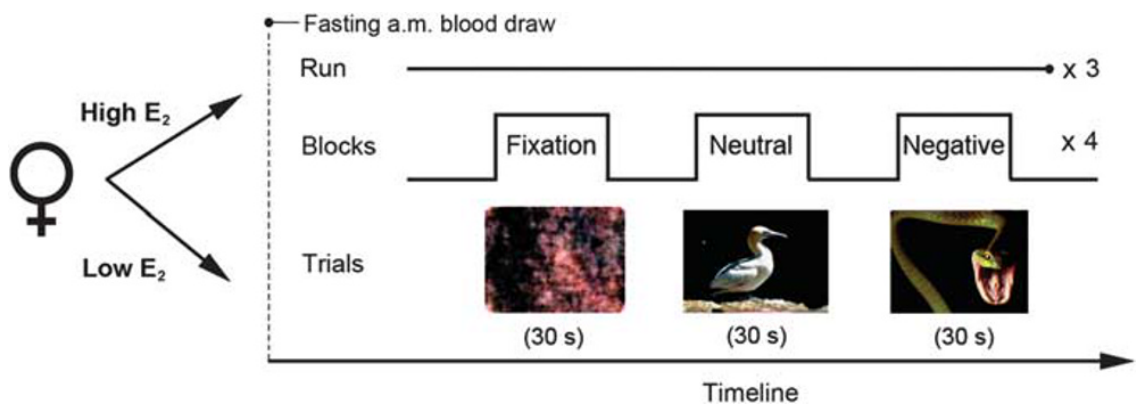

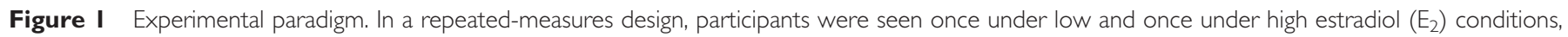

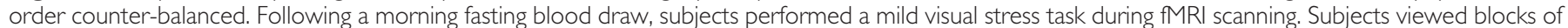

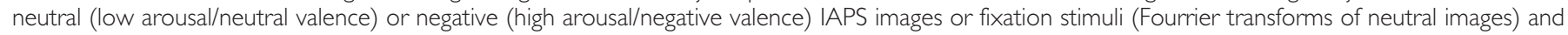

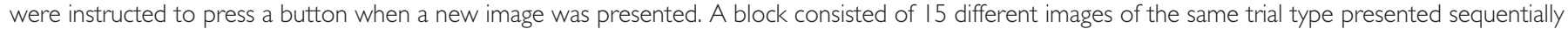
every 2 s. A run consisted of 12 blocks, four each of negative, neutral, and fixation trials. All subjects completed three runs (6 min each). 
estradiol sessions. Within the anatomical mask, functional ROIs were then defined as $5 \mathrm{~mm}$ spheres (subcortical ROIs) and $10 \mathrm{~mm}$ spheres (cortical ROI) around peak loci. The goal of this study was to examine the hormonal regulation of the network of regions involved in the mild stress paradigm. As the locus of task-evoked activity differed between cases and controls (see Supplementary Tables S1 and S2), functional ROIs were defined separately for the two groups. This allowed us to examine the hormonal regulation of task-related activity for each group, but not betweengroups, as extracted beta values were sourced from nonidentical volumes. Thus, using the functionally defined (anatomically constrained) ROIs specific to each group, we examined whether endogenous fluctuations in gonadal hormones impacted task-evoked activity. Mean beta weights from the ROIs were extracted via the REX toolbox (Whitfield-Gabrieli, 2009) and in SPSS, using repeatedmeasures ANOVA and paired-sample $t$-tests, compared as a function of hormone status (low, high estradiol) and case status (case, control). ANCOVAs were used to assess the effects of estradiol after partialling out variance attributable to progesterone plasma levels.

Behavioral data were analyzed using SPSS (version 19; SPSS Inc., Chicago, IL) using independent $t$-tests (demographic and clinical characteristics), paired sample $t$-tests (hormonal comparisons across session), and repeatedmeasures ANOVAs (mood and anxiety ratings by group and session). A $p<0.05$ was designated for statistical significance.

\section{RESULTS}

\section{Demographic and Clinical Characteristics}

Table 1 reports demographic and clinical characteristics of the MDD and healthy control women, who were comparable on body mass index, education, parental socioeconomic status, and estimated full scale IQ. MDD women (47.4 years) were marginally older than controls (45.2 years); however, no participant was postmenopausal at the time of scanning based on menstrual cycle histories and scan-day pituitary and gonadal hormone profiles (see Materials and Methods).

\section{Hormonal Evaluations}

Fasting morning serum concentrations of HPG axis hormones in healthy controls were as follows (mean, SD): $17 \beta$-estradiol $(82.4,58.7 \mathrm{pg} / \mathrm{ml})$; progesterone $(2.0,2.9 \mathrm{ng} / \mathrm{ml})$; testosterone (30.3, $15.4 \mathrm{ng} / \mathrm{dl}) ; \mathrm{LH}(7.7,10.3 \mathrm{mIU} / \mathrm{ml}) ; \mathrm{FSH}$ $(8.5,5.1 \mathrm{mIU} / \mathrm{ml})$; and SHBG $(57.4,26.2 \mathrm{nmol} / \mathrm{l})$. Hormone values for MDD cases were as follows (mean, SD): $17 \beta$ estradiol $(103.5,91.6 \mathrm{pg} / \mathrm{ml})$; progesterone $(1.9,2.5 \mathrm{ng} / \mathrm{ml})$; testosterone (27.9, $15.7 \mathrm{ng} / \mathrm{dl}) ; \mathrm{LH}$ (11.4, $9.7 \mathrm{mIU} / \mathrm{ml}) ; \mathrm{FSH}$ $(15.5,16.9 \mathrm{mIU} / \mathrm{ml})$; and SHBG $(48.2,23.8 \mathrm{nmol} / \mathrm{l})$. Participants' scan days were categorized as 'low' or 'high' estradiol (henceforth 'low $\mathrm{E}_{2}$ ' and 'high $\mathrm{E}_{2}$ ') based on the relative change in $17 \beta$-estradiol between the two test sessions. All women included in the analyses exhibited $a \geqslant 25 \%$ change in estradiol (mean: 276.4\%) between visits. A two-way ANOVA confirmed that estradiol values differed significantly between visits $(\mathrm{F}(1,42)=20.61, p<0.001)$ and did not differ between cases and controls $(p=0.26)$. Progesterone
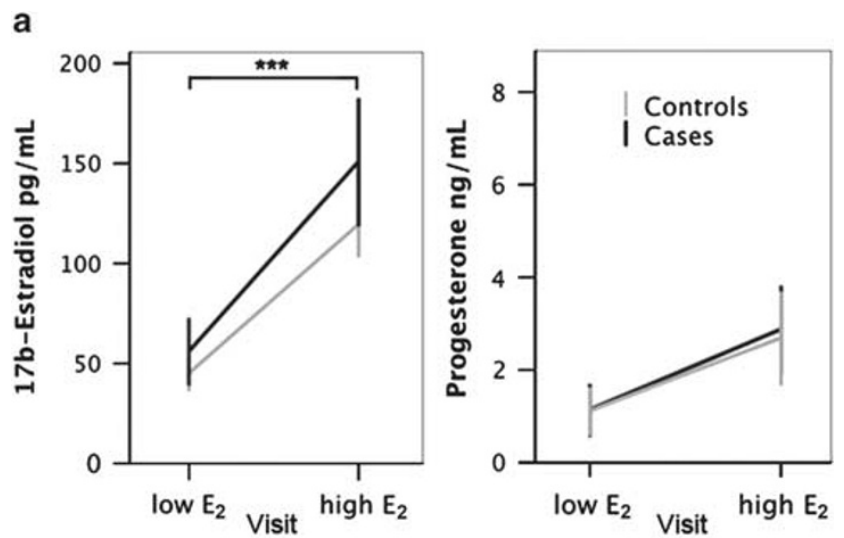

b
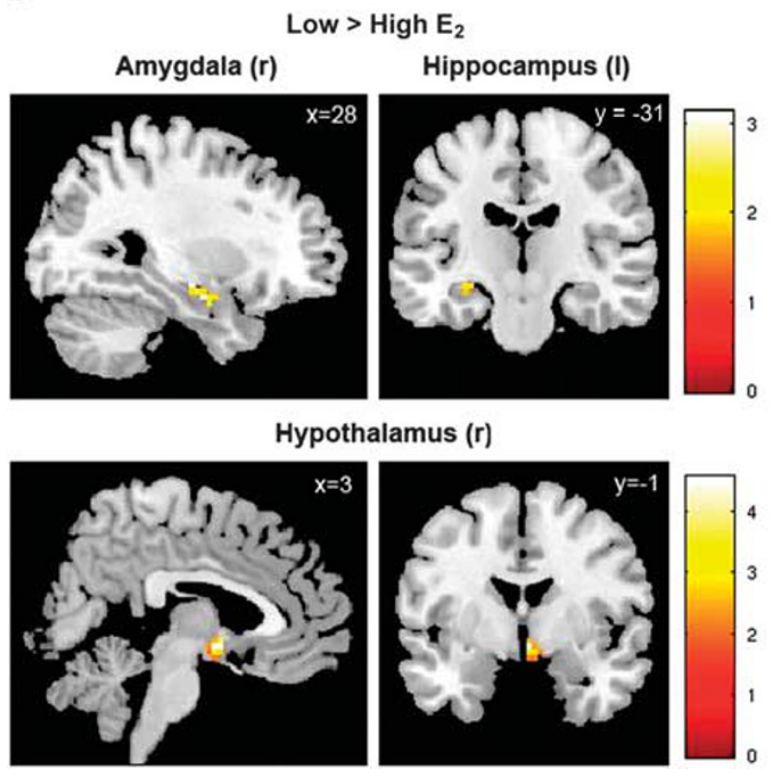

Figure 2 Estradiol attenuates stress circuitry activity in healthy women. (a) Morning fasting serum 17 $\beta$-estradiol and progesterone concentrations in healthy controls and MDD cases during the 'low $E_{2}$ ' and 'high $E_{2}$ ' visit. Estradiol values differed significantly between the two sessions $(p<0.00 \mathrm{I})$ and did not differ between cases and controls $(p=0.26)$. Progesterone levels were marginally different between visits $(p=0.043)$ but not between groups $(p=0.89)$. Error bars represent SE. (b) In healthy controls, statistical parametric maps within anatomical ROls illustrate greater task-related $\mathrm{BOLD}$ response (negative $>$ neutral stimuli) in amygdala, hippocampus, and hypothalamus under low vs high $E_{2}$ conditions. Note that these maps are for display purposes only and were not used to quantify differences in beta estimates for ROI analyses (see Supplementary Tables SI and S2 and Supplementary Figure SI).

levels were marginally different between visits $(\mathrm{F}(1,42)=$ 4.33, $p=0.04)$ but not between groups $(p=0.89)$ (Figure 2a). The between-visit difference in progesterone levels did not reach significance in post-hoc paired-sample $t$-tests stratified by case status (controls, $p=0.17$; cases, $p=0.22$ ), although this could be attributable to the reduction in degrees of freedom. Serum concentrations of additional HPG axis hormones, including FSH, testosterone, SHBG, and the computed free androgen index (FAI) are summarized in Supplementary Figure S1. Two-way ANOVAs indicated that FSH values did not differ between between visits $(\mathrm{F}(1,42)=2.36, p=0.13)$ but did differ marginally by 
group $(\mathrm{F}(1,42)=4.05, p=0.05)$; testosterone levels did not differ by visit $(p=0.25)$ or group $(p=0.61)$; SHBG did not differ by visit $(p=0.77)$ or group $(p=0.24)$; and FAI, which provides an estimate of 'free' testosterone concentrations, did not differ by visit $(p=0.31)$ or group $(p=0.87)$. Finally, no group by visit interaction reached significance. To isolate the potential effects of $17 \beta$-estradiol on task-related BOLD, we included progesterone plasma levels as a covariate in subsequent ANCOVAs. Below we report results with and without partialing out variance attributable to progesterone level. Results did not differ appreciably between models.

\section{Mood and Anxiety Ratings}

As expected, MDD women reported higher STAI trait anxiety compared with controls $(\mathrm{F}(1,40)=28.48, p<0.001)$. However, mean levels in the MDD group were below the clinical range for older adults (see Table 2), consistent with a remitted status. A repeated-measures ANOVA comparing prescan and postscan state anxiety revealed a main effect of case $(\mathrm{F}(1,39)=12.54, p=0.001)$, with MDD women reporting overall higher state anxiety. There was a trend toward a case by time interaction $(\mathrm{F}(1,39)=3.36, p=0.07)$ in which cases reported a greater increase in state anxiety following the visual stress paradigm. MDD women also reported higher postscan scores on the Tension-Anxiety $(p<0.02)$, Depression-Dejection $(p<0.04)$, and Fatigue-Inertia $(p<0.005)$ subscales of the POMS (Table 2$)$. Anxiety and mood scores did not differ as a function of estradiol status. Subjective ratings of task stimuli did not differ between cases and controls or as a function of estradiol status. Collectively, these findings suggest a somewhat more marked behavioral response to the mild stress challenge in MDD women, with higher anxiety and increased negative mood after the challenge, consistent with a lower threshold for stress reactivity despite equivalent subjective stimuli ratings as control women (Holsen et al 2013).

\section{FMRI Visual Stress Challenge}

The mild visual stress challenge evoked robust bilateral responses in cortical (mPFC) and subcortical (amygdala, hippocampus, and hypothalamus) regions known to have a critical role in the appraisal and regulation of the stress response (Supplementary Figure S2). Supplementary Tables S1 and S2 report regions of BOLD signal intensity values (negative-neutral stimuli) for cases and controls based on 'supergroup' activity within a priori anatomical ROIs (defined at $p_{\text {FWE }}<0.05$, small volume-corrected). 'Supergroup' maps represent activity averaged across participants' two scanning sessions (ie, low and high $\mathrm{E}_{2}$ scans) and thus were agnostic to estradiol status so as not to bias subsequent analyses. Task-induced activation was evident throughout multiple stress circuitry regions (Supplementary Figure S2).

Under low estradiol conditions, healthy controls showed robust task-related BOLD activity (negative $v s$ neutral stimuli) across subcortical ROIs, including bilateral amygdala, hippocampus, and hypothalamus. Under elevated estradiol conditions, activity in these regions was significantly diminished (paired-sample $t$-tests, low $>$ high estradiol, right amygdala $(p=0.003)$; left hippocampus $(p=0.029)$; right hypothalamus $(p=0.011))$. Trend-level differences were observed in the left amygdala $(p=0.074)$ and left hypothalamus $(p=0.062)$ (Figure 3a). Next, we determined whether the impact of estradiol remained after partialing out variance attributable to progesterone levels, which also varied across the menstrual cycle. Results did not change appreciably: low $>$ high estradiol, right amygdala, (ANCOVA, $\left.\mathrm{F}(1,23)=6.7, p=0.017, R^{2}=0.25\right)$; left amygdala, $\left(\mathrm{F}(1,23)=3.2\right.$, trend $\left.p=0.085, R^{2}=0.13\right)$; left hippocampus $\left(\mathrm{F}(1,23)=4.9, \quad p=0.037, R^{2}=0.18\right)$; right hypothalamus $\left(\mathrm{F}(1,23)=5.9, p=0.024, R^{2}=0.23\right) ;$ and left hypothalamus $\left(\mathrm{F}(1,23)=6.7, p=0.016, R^{2}=0.27\right)$. Within MDD women, this hormonal regulation of stress circuitry was absent. No significant BOLD changes in stress circuitry ROIs were evident between low and high estradiol sessions ( $p>0.1$ for all ROIs), and results did not change after modeling the additional set of sex hormones ( $p>0.1$ for all ROIs) (Figure 3b).

\section{DISCUSSION}

This study demonstrates that the ovarian hormone $17 \beta$ estradiol regulates neural activity within key subcortical regions in stress circuitry, including the amygdala, hippocampus, and hypothalamus, in response to a mild visual stress challenge. In healthy women, there were robust taskevoked BOLD activations in these stress circuitry regions under low estradiol conditions, which were attenuated when estradiol levels were naturally elevated. These results extend our previous work in healthy women, which found that BOLD activity in cortical and subcortical stress response regions was attenuated during the late follicular/midcycle phase compared with early follicular phase of the menstrual cycle (Goldstein et al, 2005). The present study suggests that this modulation of stress circuitry activity is due to endogenous fluctuations in $17 \beta$-estradiol. Further, we show that the hormonal capacity to regulate task-evoked neural activity was absent in remitted MDD women.

A substantial amount of work has been conducted within basic endocrinology studies to characterize the interaction between the HPA and HPG axes. Based on early endocrine studies in rodents, estradiol was thought to enhance stress responsivity by potentiating basal and stimulated levels of adrenocorticotropic hormone and corticosterone (Viau and Meaney, 1991; Burgess and Handa, 1992; Carey et al, 1995). More recent evidence suggests that estradiol's impact is dose and receptor dependent (ie, $\mathrm{ER} \alpha$ and $\mathrm{ER} \beta$ ) (Lund et al, 2005; Weiser et al, 2008). Low-dose, short-term estradiol administration can inhibit HPA axis reactivity (Redei et al, 1994; Young et al, 2001; Dayas et al, 2000; Komesaroff et al, 1998), whereas higher doses and long-term administration can enhance HPA axis responses (Burgess and Handa, 1992; Carey et al, 1995; Viau and Meaney, 1991; Schmidt and Rubinow, 2009). Further, estradiol may have opposing effects on HPA axis reactivity depending on the ER subtype being targeted, with excitatory effects of the steroid hormone at $\operatorname{ER} \alpha$ and inhibitory effects at $\operatorname{ER} \beta$ (Lund et al, 2005, 2006; Oyola et al, 2012; Weiser et al, 2010). Our findings build on this literature by using functional neuroimaging to demonstrate that estradiol modulates the activity of brain regions responsible for regulating HPA axis activity. Importantly, our data do not rule out the possibility 
Table 2 Mood and Anxiety Ratings in Healthy and MDD Women by Estradiol Session

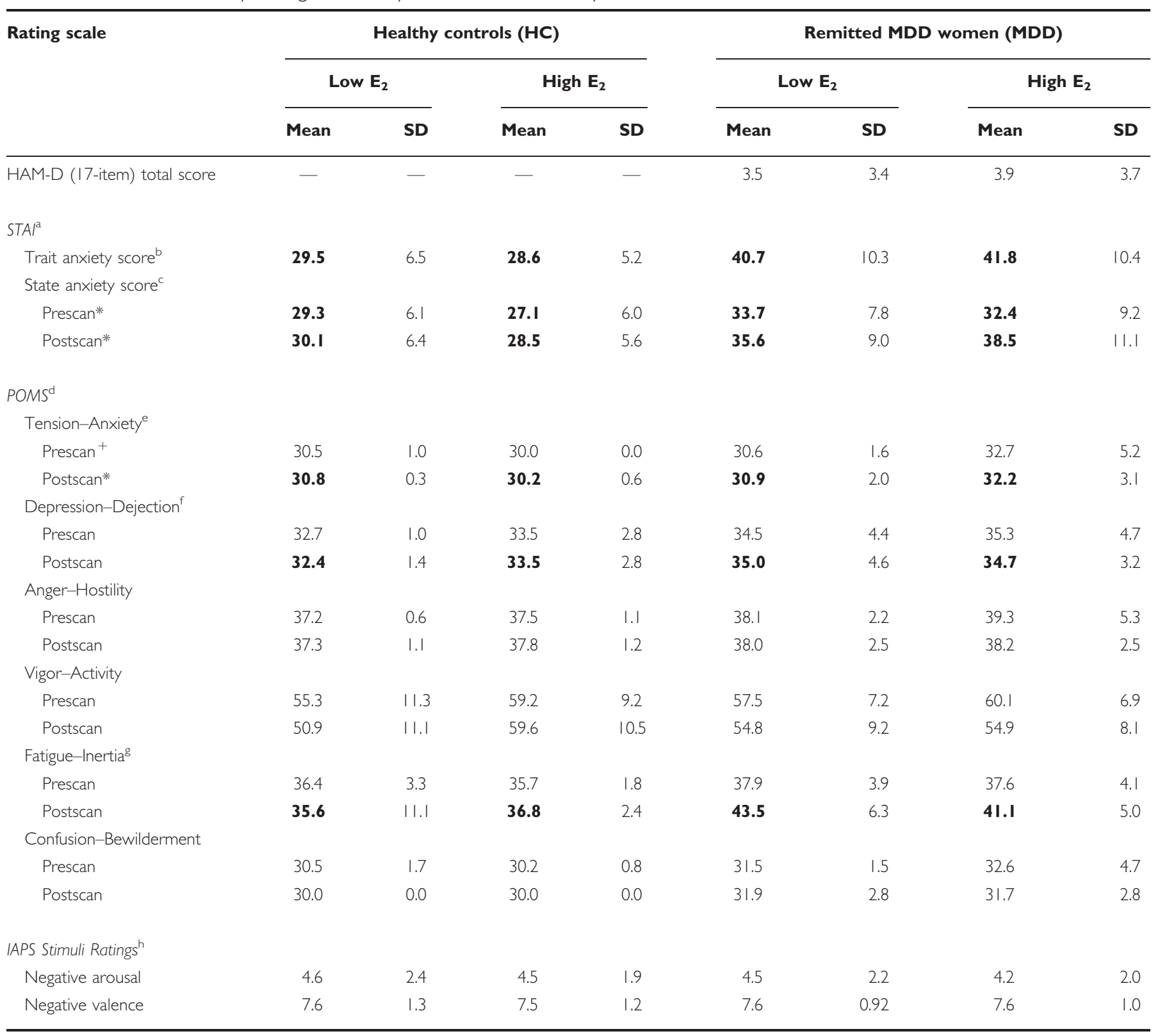

aSpielberger State-Trait Anxiety Inventory (STAI) is a self-report of current and "usual" levels of anxiety.

${ }^{\mathrm{b}}$ Trait anxiety, MDD $>\mathrm{HC}, p<0.001$.

'State anxiety, MDD $>\mathrm{HC}, p=0.013$ (prescan), $p<0.00$ I (postscan). Repeated-measures ANOVA revealed that MDD cases reported a bigger increase in state anxiety after the visual stress paradigm compared with baseline values $(F(I, 39)=3.36$, trend $p=0.074$.

dProfile of Mood States (POMS) is a self-report of current mood states.

${ }^{\mathrm{e}} \mathrm{TA}, \mathrm{MDD}>\mathrm{HC}$, trend $p=0.095$ (prescan), MDD $>\mathrm{HC}, p=0.018$ (postscan).

fDD, MDD $>\mathrm{HC}, p=0.037$ (postscan).

${ }^{\mathrm{F}} \mathrm{Fl}, \mathrm{MDD}>\mathrm{HC}, \mathrm{p}=0.003$ (postscan).

${ }^{h}$ After the $\mathrm{fMRI}$ scanning session, subjects rated a selection of the International Affective Picture System (IAPS) stimuli on valence and arousal. Bold values indicate a significant different $(P<0.05)$ between groups.

that additional gonadal hormones, including progesterone and its neurosteroid metabolites, contribute to the observed effects. Although we included progesterone levels in our ANCOVA model, this cannot fully account for its presence. Indeed, there is substantial evidence that progesterone and/or its neuroactive metabolites regulate HPA axis function (Roca et al, 2003) and have a role in the development of mood disorders, including postpartum depression (Bloch et al, 2000).

Additional studies from our group and others have found menstrual-cycle dependent changes in amygdala, hippocampal, and/or mPFC activity in response to psycho- 
a

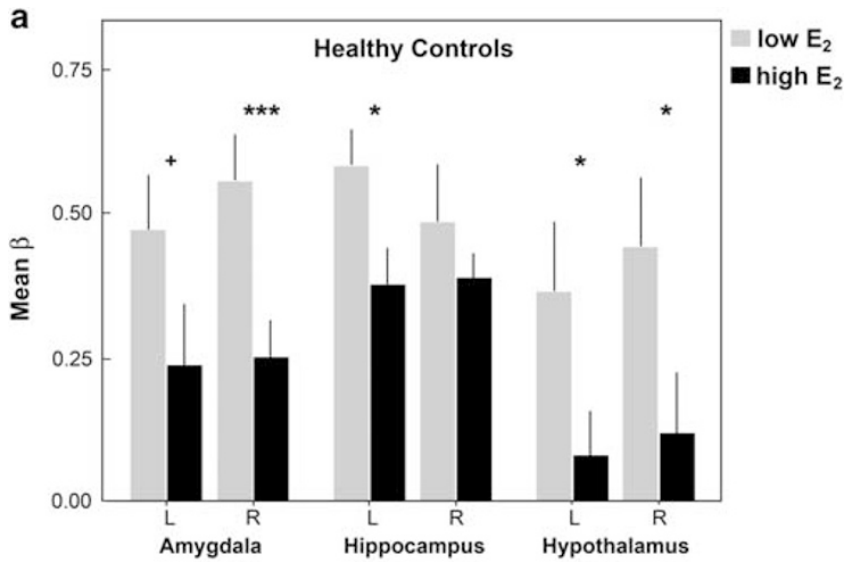

b

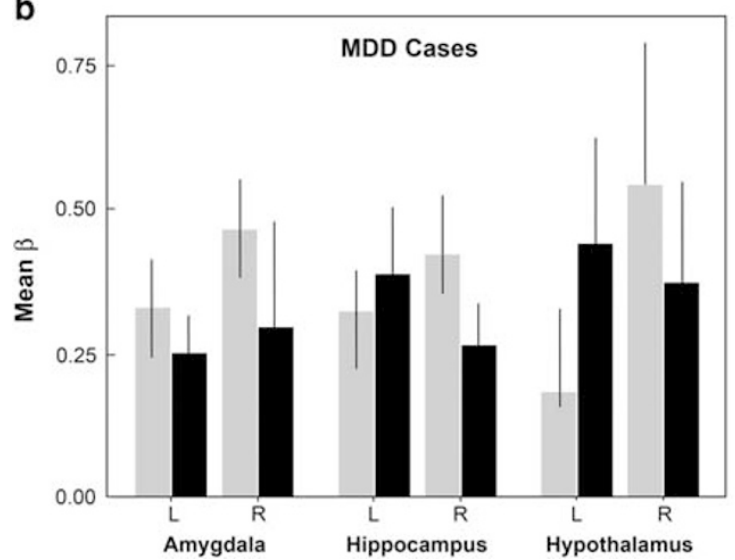

Figure 3 Estradiol attenuates task-evoked stress circuitry activity in healthy women. This hormonal regulation was not observed in MDD women. Parameter estimates were determined from functional ROls defined at the 'supergroup' level, agnostic to estradiol status. Beta values are displayed for bilateral amydala, hippocampus, and hypothalamus ROls in healthy controls (a) and MDD cases (b). Values reflect raw beta estimates unadjusted for progesterone. $* p<0.05, * * *<0.01$, $* * * * * 0.005$. Error bars represent SE.

logical stress (Ossewaarde et al, 2010) and negative images (Goldstein et al, 2005; Andreano and Cahill, 2010), providing compelling indirect evidence that gonadal hormones have a role in shaping stress circuitry function. Our results extend this literature by demonstrating, in vivo in humans, that $17 \beta$-estradiol regulates activity across key stress response regions. Our findings are consistent with other neuroimaging studies that report gonadal hormoneor menstrual cycle-dependent changes in neural activity within working memory, arousal, and reward circuitries (Berman et al, 1997; Shaywitz et al, 1999; Goldstein et al, 2005; Dreher et al, 2007; Van Wingen et al, 2008; Jacobs and D'Esposito, 2011). Recent evidence also demonstrates that exogenously administered sex steroids alter regional glucose metabolism in a formulation-dependent manner (Rasgon et al, 2014). Collectively, these findings underscore the diversity of conditions under which gonadal hormones shape brain function. Importantly, the effects of estradiol we observed were condition and region specific and not driven by global changes in blood oxygenation.

Further, these data provide evidence that estradiol's ability to modulate stress circuitry is dysregulated in women with MDD. Strikingly, these findings were present in MDD women despite their fully or partially remitted status, suggesting enduring changes that persist beyond acute clinical episodes. Given that serum estradiol concentrations were indistinguishable between cases and controls, our findings raise the possibility that ER expression is downregulated in stress circuitry regions in MDD women, thereby blunting estradiol's actions. This idea is consistent with evidence of glucocorticoid receptor downregulation in the hippocampus and mPFC during chronic stress (Herman et al, 1995) and in MDD individuals (Webster et al, 2002), resulting in reduced glucocorticoid negative feedback sensitivity and, in turn, an inability to regulate HPA axis reactivity (Mizogushi et al, 2003). In fact, in a postmortem analysis of the PFC, hippocampus, and amygdala of MDD cases and matched healthy controls (all mid-life adults), the expression of $\mathrm{ER} \alpha \mathrm{mRNA}$ transcripts was markedly different between groups, with MDD women expressing less ER $\alpha$ mRNA in the PFC and amygdala (Perlman et al, 2005). Similarly, Gerrits et al (2005) found that chronic stress altered ER expression in the paraventricular nucleus (PVN) of the hypothalamus, a region that contains the densest level of corticotropin-releasing hormone $(\mathrm{CRH})$ neurons in the brain and is critically important for controlling the cascade of pituitary and adrenal stress hormones (Stratton et al, 2011; Zuloaga et al, 2012). Postmortem analyses have also revealed that $\mathrm{CRH}$-producing neurons co-localize with $\mathrm{ER} \alpha$ in the $\mathrm{PVN}$ (Bao et al, 2005). $\mathrm{ER} \beta$ is also expressed in the PVN (Weiser et al, 2008). The present findings in healthy women indirectly implicate the PVN by demonstrating estradiol-driven BOLD activity changes in the hypothalamus. Taken together, differences in ER expression between cases and controls in key stress circuitry regions may lead to chronic signalling deficits, an effect that could underlie our observed effects at the level of BOLD activity.

This study helps advance our understanding of the neurobiological mechanisms underlying the marked sex difference in MDD risk by demonstrating that $17 \beta$-estradiol impacts intermediate phenotypes of MDD, represented here as dysregulated brain activity in stress response circuitry. Although deficits in HPA axis function are a hallmark of MDD (Gibbons and McHugh, 1962; Carroll et al, 1976a, b; Nemeroff et al, 1984; Arborelius et al, 1999; Webster et al, 2002; Parker et al, 2003; Holsen et al, 2013), gonadal hormone deficits are also evident (Rubinow and Schmidt, 1996, 2006; Young et al, 2000, 2007; Himelein and Thatcher, 2006; Young and Korszun, 2002; Graziottin and Serafini, 2009; for a review, see Goldstein et al, 2014). For example, women with persistent MDD have twice the risk of early perimenopausal transition. That is, women with a lifetime history of MDD were found to have higher FSH and lower estradiol levels, indicative of early decline in ovarian function (Young et al, 2000; Harlow et al, 2003), and some reports indicate that ovarian dysfunction procedes the onset of MDD (Harlow et al, 2003). Our findings extend this literature by suggesting that gonadal hormone abnormalities present in MDD women have measurable consequences at the level of the brain. Although serum estradiol (and progesterone) levels were indistinguishable between cases and controls in our sample, there were clear deficits in MDD women in estradiol's ability to regulate stress 
circuitry activity in response to even a mild stress challenge. These findings may provide insights into the neural mechanisms that contribute to the established sex difference in MDD risk.

Several factors may contribute to our observation that estradiol failed to influence stress circuitry activity in MDD women. First, estradiol's ability to regulate neural activity in stress circuitry may be blunted in MDD, a finding that was present in fully or partially remitted cases and thus may reflect a trait characteristic. Second, the MDD women in our sample were slightly older than controls. It is unlikely that age can wholly explain our findings given that serum estradiol and progesterone levels did not differ between cases and controls (Figure 1) and the age difference, while significant, was minimal ( $\sim 2$ years). However, given the age range of our sample (43-50 years), slightly older ages among MDD women may have resulted in higher likelihood of perimenopausal status among the MDD cases. This is supported by previous reports that MDD women are at an increased risk of early ovarian decline (Young et al, 2000; Harlow et al, 2003) and our finding of higher FSH levels in cases (marginally significant). Third, half of the remitted MDD cases (55\%) were on psychotropic medication at the time of scanning. These medications can directly or indirectly alter fMRI BOLD responses and could obscure an estradiol-driven difference in BOLD. For example, SSRI administration has been shown to decrease BOLD activity in the amygdala in MDD patients (Godlewska et al, 2012) and alter medial PFC-hippocampal functional connectivity in healthy controls (McCabe et al, 2011). Thus it is impossible to formally rule out the potential confounding effects of medication given that the present study was not powered to analyze MDD data by medication status. However, histogram plots depicting mean beta values across each brain region of interest suggest that medicated and unmedicated cases had highly overlapping BOLD responses (Supplementary Figure S3). Note, though, that MDD patients displayed a higher degree of variance in BOLD (particularly within the hypothalamus), which could be driven by medication status or comorbidities.

Although our sample size in general was not large, we designed a within-person repeated-measures protocol that increased the statistical power of the study, with each woman serving as her own control. Using this approach, we demonstrated an impact of estradiol on BOLD changes in multiple subcortical regions that are involved in the regulation of stress circuitry and contain the highest levels of sex steroid receptors in the brain (ie, hypothalamus, amygdala, and hippocampus). It is possible that additional stress circuitry regions, for example, $\mathrm{mPFC}$, are also regulated by $17 \beta$-estradiol as they express ER receptors (albeit at a lower level). However, we did not observe such effects, which may be attributable to insufficient statistical power and thus needs replication.

In conclusion, the findings reported here contribute to identifying the neural substrates associated with the longstanding clinical observation that changes in women's reproductive system are related to mood fluctuations and MDD symptomology (Rabin et al, 1990; Baischer et al, 1995; Rubinow and Schmidt, 1996; Harlow et al, 2003; Payne, 2003; Roca et al, 2003; Spinelli, 2005; Payne et al, 2009). Using a mild visual stress fMRI paradigm that reliably activates stress circuitry, we demonstrated a role for estradiol in modulating stress circuitry activity in healthy women and found evidence for the disruption of this hormone-brain pathway in major depression. Taken together, these results will contribute to our broader understanding of the impact of sex steroid hormones on the expression of mood and anxiety traits as well as sex differences in the risk for MDD.

\section{FUNDING AND DISCLOSURE}

This study was supported by the Office for Research on Women's Health (ORWH) and National Institute of Mental Health (ORWH-NIMH SCOR P50 MH082679; Goldstein, Tobet, Handa, PIs). Dr Jacobs was supported by the NIMH T32 MH016259 and the NICHD (BIRCWH) K12 HD051959. The authors declare no conflict of interest.

\section{ACKNOWLEDGEMENTS}

We thank Harlyn Aizley, M.Ed. for administering structured clinical interviews and Stuart Tobet, Ph.D. and Robert Handa, Ph.D. for earlier insightful comments on this work.

\section{REFERENCES}

Andreano JM, Cahill L (2010). Menstrual cycle modulation of medial temporal activity evoked by negative emotion. Neuroimage 53: 1286-1293.

Angold A, Costello EJ (2006). Puberty and depression. Child Adolesc Psychiatr Clin N Am 15: 919-937, ix.

Arborelius L, Owens MJ, Plotsky PM, Nemeroff CB (1999). The role of corticotropin-releasing factor in depression and anxiety disorders. J Endocrinol 160: 1-12.

Baischer W, Koinig G, Hartmann B, Huber J, Langer G (1995). Hypothalamic-pituitary-gonadal axis in depressed premenopausal women: elevated blood testosterone concentrations compared to normal controls. Psychoneuroendocrinology 20: 553-559.

Bao AM, Hestiantoro A, Van Someren EJ, Swaab DF, Zhou JN (2005). Colocalization of corticotropin-releasing hormone and oestrogen receptor-alpha in the paraventricular nucleus of the hypothalamus in mood disorders. Brain 128: 1301-1313.

Berman KF, Schmidt PJ, Rubinow DR, Danaceau MA, Van Horn JD, Esposito G et al (1997). Modulationofcognitionspecific cortical activity by gonadal steroids: a positron-emission tomography study in women. Proc Natl Acad Sci USA 94: 8836-8841.

Bloch M, Schmidt PJ, Danaceau M, Murphy J, Nieman L, Rubinow DR (2000). Effects of gonadal steroids in women with a history of postpartum depression. Am J Psychiatry 157: 924-930.

Bradley MM, Cuthbert BN, Lang PJ (1996). Picture media and emotion: effects of a sustained affective context. Psychophysiology 33: 662-670.

Brummelte S, Galea LA (2010). Depression during pregnancy and postpartum: contribution of stress and ovarian hormones. Prog Neuropsychoph 34: 766-776.

Burgess LH, Handa RJ (1992). Chronic estrogen-induced alterations in adreno- corticotropin and corticosterone secretion, and glucocorticoid receptor-medi- ated functions in female rats. Endocrinology 131: 1261-1269.

Carey MP, Deterd CH, de Koning J, Helmerhorst F, De Kloet ER (1995). The influence of ovarian steroids on hypothalamicpituitary-adrenal regulation in the female rat. J Endocrinol 144: 311-321. 
Carroll BJ, Curtis GC, Mendels J (1976a). Cerebrospinal fluid and plasma free cortisol concentrations in depression. Psychol Med 6: 235-244.

Carroll BJ, Curtis GC, Davies BM, Mendels J, Sugerman AA (1976b). Urinary free cortisol excretion in depression. Psychol Med 6: 43-50.

Clark AS, MacLusky NJ, Goldman-Rakic PS (1988). Androgen binding and metabolism in the cerebral cortex of the developing rhesus monkey. Endocrinology 123: 932-940.

Daly RC, Danaceau MA, Rubinow DR, Schmidt PJ (2003). Concordant restoration of ovarian function and mood in perimenopausal depression. Am J Psychiatry 160: 1842-1846.

Dayas CV, Xu Y, Buller KM, Day TA (2000). Effects of chronic oestrogen replacement on stress-induced activation of hypothalamic-pituitary-adrenal axis control pathways. J Neuroendocrinol 12: 784-794.

Donahue JE, Stopa EG, Chorsky RL, King JC, Schipper HM, Tobet SA et al (2000). Cells containing immunoreactive estrogen receptor-alpha in the human basal forebrain. Brain Res 856: 142-151.

Dreher JC, Schmidt PJ, Kohn P, Furman D, Rubinow D, Berman KF (2007). Menstrual cycle phase modulates reward-related neural function in women. Proc Natl Acad Sci USA 104: 2465-2470.

Freeman EW, Sammel MD, Boorman DW, Zhang R (2014). Longitudinal pattern of depressive symptoms around natural menopause. JAMA Psychiatry 71: 36-43.

Gibbons JL, McHugh PR (1962). Plasma cortisol in depressive illness. J Psychiatr Res 1: 162-171.

Goldstein JM, Jerram M, Abbs B, Whitfield-Gabrieli S, Makris N (2010). Sex differences in stress response circuitry activation dependent on female hormonal cycle. J Neurosci 30: 431-438.

Goldstein JM, Jerram M, Poldrack R, Ahern T, Kennedy DN, Seidman LJ et al (2005). Hormonal cycle modulates arousal circuitry in women using functional magnetic resonance imaging. J Neurosci 25: 9309-9316.

Goldstein JM, Handa RJ, Tobet SA (2014). Disruption of fetal hormonal programming (prenatal stress) implicates shared risk for sex differences in depression and cardiovascular disease. Front Neuroendocrinol 35: 140-158.

Gerrits M, Grootkarijn A, Bekkering BF, Bruinsma M, Boer JAD, Horst GJT (2005). Cyclic estradiol replacement attenuates stressinduced c-Fos expression in the PVN of ovariectomized rats. Brain Res Bull 67: 147-155.

Godlewska BR, Norbury R, Selvaraj S, Cowen PJ, Harmer CJ (2012). Short-term SSRI treatment normalises amygdala hyperactivity in depressed patients. Psychol Med 42: 2609-2617.

Graziottin A, Serafini A (2009). Depression and the menopause: why antidepressants are not enough? Menopause Int 15: 76-81.

Handa RJ, Burgess LH, Kerr JE, O’Keefe JA (1994). Gonadal steroid hormone receptors and sex differences in the hypothalamopituitary-adrenal axis. Horm Behav 28: 464-476.

Hao J, Rapp PR, Leffler AE, Leffler SR, Janssen WG, Lou W et al (2006). Estrogen alters spine number and morphology in prefrontal cortex of aged female rhesus monkeys. J Neurosci 26: $2571-2578$.

Harlow BL, Wise LA, Otto MW, Soares CN, Cohen LS (2003). Depression and its influence on reproductive endocrine and menstrual cycle markers associated with perimenopause: the Harvard Study of Moods and Cycles. Arch Gen Psychiatry 60: 29-36.

Herman JP, Adams D, Prewitt CM (1995). Regulatory changes in neuroendocrine stress-integrative circuitry produced by a variable stress paradigm. Neuroendocrinology 61: 180-190.

Himelein MJ, Thatcher SS (2006). Polycystic ovary syndrome and mental health: a review. Obstet Gynecol Surv 61: 723-732.

Holsen LM, Spaeth SB, Lee JH, Ogden LA, Klibanski A, WhitfieldGabrieli S et al (2011). Stress response circuitry hypoactivation related to hormonal dysfunction in women with major depression. J Affect Disord 131: 379-387.
Holsen LM, Lancaster K, Klibanski A, Whitfield-Gabrieli S, Cherkerzian S, Buka S et al (2013). HPA-axis hormone modulation of stress response circuitry activity in women with remitted major depression. Neuroscience 250: 733-742.

Jacobs E, D'Esposito M (2011). Estrogen shapes dopaminedependent cognitive processes: implications for women's health. J Neurosci 31: 5286-5293.

Kawata M (1995). Roles of steroid hormones and their receptors in structural organization in the nervous system. Neurosci Res 24: $1-46$.

Komesaroff PA, Esler M, Clarke IJ, Fullerton MJ, Funder JW (1998). Effects of estrogen and estrous cycle on glucocorticoid and catecholamine responses to stress in sheep. Am J Physiol 275: E671-E678.

Lund TD, Rovis T, Chung WC, Handa RJ (2005). Novel actions of estrogen receptor- $\beta$ on anxiety-related behaviors. Endocrinology 146.2: 797-807.

Lund TD, Hinds LR, Handa RJ (2006). The androgen 5alphadihydrotestosterone and its metabolite 5alpha-androstan-3beta, 17beta-diol inhibit the hypothalamo-pituitary-adrenal response to stress by acting through estrogen receptor beta-expressing neurons in the hypothalamus. J Neurosci 26: 1448-1456.

MacLusky NJ, Clark AS, Naftolin F, Goldman-Rakic PS (1987). Estrogen formation in the mammalian brain: possible role of aromatase in sexual differentiation of the hippocampus and neocortex. Steroids 50: 459-474.

Maldjian JA, Laurienti PJ, Kraft RA, Burdette JH (2003). An automated method for neuroanatomic and cytoarchitectonic atlasbased interrogation of fMRI data sets. Neuroimage 19: 1233-1239.

Makris N, Swaab DF, van der Kouwe A, Abbs B, Boriel D, Handa RJ et al (2013). Volumetric parcellation methodology of the human hypothalamus in neuroimaging: normative data and sex differences. Neuroimage 69: 1-10.

McCabe C, Mishor Z, Filippini N, Cowen PJ, Taylor MJ, Harmer CJ (2011). SSRI administration reduces resting state functional connectivity in dorso-medial prefrontal cortex. Mol Psychiatry 16: $592-594$.

Mizoguchi K, Ishige A, Aburada M, Tabira T (2003). Chronic stress attenuates glucocorticoid negative feedback: involvement of the prefrontal cortex and hippocampus. Neuroscience 119: 887-897.

Morrison MF, Kallan MJ, Ten Have T, Katz I, Tweedy K, Battistini $M$ (2004). Lack of efficacy of estradiol for depression in postmenopausal women: a randomized, controlled trial. Biol Psychiatry 55: 406-412.

Nabekura J, Oomura Y, Minami T, Mizuno Y, Fukuda A (1986). Mechanism of the rapid effect of 17 beta-estradiol on medial amygdala neurons. Science 233: 226-228.

Nemeroff CB, Widerlov E, Bissette G, Walleus H, Karlsson I, Eklund $\mathrm{K}$ et al (1984). Elevated concentrations of CSF corticotropin-releasing factor-like immunoreactivity in depressed patients. Science 226: 1342-1344.

Nikolarakis KE, Almeida OFX, Herz A (1986). Corticotropinreleasing factor (CRF) inhibits gonadotropin-releasing hormone $(\mathrm{GnRH})$ release from superfused rat hypothalami in vitro. Brain Res 377: 388-390.

Niswander KR, Gordon M (1972). The Women and Their Pregnancies: The Collaborative Perinatal Study of the National Institute of Neurological Diseases and Stroke: U.S. Department of Health, Education, and Welfare; Government Printing Office: Washington, DC, USA.

Ossewaarde L, Hermans EJ, van Wingen GA, Kooijman SC, Johansson IM, Bäckström T et al (2010). Neural mechanisms underlying changes in stress-sensitivity across the menstrual cycle. Psychoneuroendocrinology 35: 47-55.

Östlund H, Keller E, Hurd YL (2003). Estrogen receptor gene expression in relation to neuropsychiatric disorders. Ann NY Acad Sci 1007: 54-63. 
Österlund MK, Gustafsson JA, Keller E, Hurd YL (2000). Estrogen receptor $\beta$ (ER $\beta)$ messenger ribonucleic acid (mRNA) expression within the human forebrain: distinct distribution pattern to ER $\alpha$ mRNA 1. J Clin Endocrinol Metab 85: 3840-3846.

Österlund MK, Hurd YL (2001). Estrogen receptors in the human forebrain and the relation to neuropsychiatric disorders. Prog Neurobiol 64.3: 251-267.

Oyola MG, Portillo W, Reyna A, Foradori CD, Kudwa A, Hinds L et al (2012). Anxiolytic effects and neuroanatomical targets of estrogen receptor-beta (ERbeta) activation by a selective ERbeta agonist in female mice. Endocrinology 153: 837-846.

Parker KJ, Schatzberg AF, Lyons DM (2003). Neuroendocrine aspects of hypercortisolism in major depression. Horm Behav 43: 60-66.

Payne JL (2003). The role of estrogen in mood disorders in women. Int Rev Psychiatry 15: 280-290.

Payne JL, Palmer JT, Joffe H (2009). A reproductive subtype of depression: conceptualizing models and moving toward etiology. Harv Rev Psychiatry 17: 72-86.

Perlman WR, Tomaskovic-Crook E, Montague DM, Webster MJ, Rubinow DR, Kleinman JE et al (2005). Alteration in estrogen receptor $\alpha$ mRNA levels in frontal cortex and hippocampus of patients with major mental illness. Biol Psychiatry 58: 812-824.

Plotsky PM, Owens MJ, Nemeroff CB (1998). Psychoneuroendocrinology of depression. Hypothalamic-pituitary-adrenal axis. Psychiatr Clin North Am 21: 293-307.

Redei E, Li L, Halasz I, McGivern RF, Aird F (1994). Fast glucocorticoid feedback inhibition of ACTH secretion in the ovariectomized rat: effect of chronic es trogen and progesterone. Neuroendocrinology 60: 113-123.

Rabin DS, Schmidt PJ, Campbell G, Gold PW, Jensvold M, Rubinow DR et al (1990). Hypothalamic-pituitary-adrenal function in patients with the premenstrual syndrome. J Clin Endocrinol Metab 71: 1158-1162.

Rasgon NL, Geist CL, Kenna HA, Wroolie TE, Williams KE, Silverman DH (2014). Prospective randomized trial to assess effects of continuing hormone therapy on cerebral function in postmenopausal women at risk for dementia. PLoS One 9: e89095.

Roca CA, Schmidt PJ, Altemus M, Deuster P, Danaceau MA, Putnam K et al (2003). Differential menstrual cycle regulation of hypothalamic-pituitary-adrenal axis in women with premenstrual syndrome and controls. J Clin Endocrinol Metab 88: 3057-3063.

Rubinow DR, Schmidt PJ (1996). Androgens, brain, and behavior. Am J Psychiatry 153: 974-984.

Rubinow DR, Schmidt PJ (2006). Gonadal steroid regulation of mood: the lessons of premenstrual syndrome. Front Neuroendocrinol 27: 210-216.

Saletu B, Brandstatter N, Metka M, Stamenkovic M, Anderer P, Semlitsch HV et al (1996). Hormonal, syndromal and EEG mapping studies in menopausal syndrome patients with and without depression as compared with controls. Maturitas 23: 91-105.

Sattler J (1992). Assessment of Children. 3rd Edition. San Diego, Jerome Sattler.

Schmidt PJ, Rubinow DR (2009). Sex hormones and mood in the perimenopause. Ann NY Acad Sci 1179: 70-85.

Schmidt PJ, Murphy JH, Haq N, Danaceau MA, Clair L St (2002). Basal plasma hormone levels in depressed per- imenopausal women. Psychoneuroendocrinology 27: 907-920.

Schmidt PJ, Nieman L, Danaceau MA, Tobin MB, Roca CA, Murphy JH et al (2000). Estrogen replacement in perimenopause- related depression: a preliminary report. Am J Obstet Gynecol 183: 414-420.

Shaywitz SE, Bennett A, Pugh KR, Fulbright RK, Skudlarksi P, Mencl WE et al (1999). Effect of estrogen on brain activation patterns in postmenopausal women during working memory tasks. JAMA 281: 1197-1202.

Spinelli MG (2005). Neuroendocrine effects on mood. Rev Endocr Metab Disord 6: 109-115.

Soares CD, Almeida OP, Joffe H, Cohen LS (2001). Efficacy of estradiol for the treatment of depressive dis- orders in perimenopausal women: a double-blind, randomized, placebocontrolled trial. Arch Gen Psychiatry 58: 529-534.

Stratton MS, Searcy BT, Tobet SA (2011). GABA regulates corticotropin releasing hormone levels in the paraventricular nucleus of the hypothalamus in newborn mice. Physiol Behav 104: 327-333.

Tobet SA, Hanna IK (1997). Ontogeny of sex differences in the mammalian hypothalamus and preoptic area. Cell Mol Neurobiol 17: $565-601$.

Ulrich-Lai YM, Herman JP (2009). Neural regulation of endocrine and autonomic stress responses. Nat Rev Neurosci 10: 397-409.

Van Wingen GA, Van Broekhoven F, Verkes RJ, Petersson KM, Bäckström T, Buitelaar JK et al (2008). Progesterone selectively increases amygdala reactivity in women. Mol Psychiatry 13: 325-333.

Viau V, Meaney MJ (1991). Variations in the hypothalamicpituitary-adrenal re- sponse to stress during the estrous cycle in the rat. Endocrinology 129: 2503-2511.

Walf AA, Frye CA (2006). A review and update of mechanisms of estrogen in the hippocampus and amygdala for anxiety and depression behavior. Neuropsychopharmacology 31: 1097-1111.

Wang AC, Hara Y, Janssen WG, Rapp PR, Morrison JH (2010). Synaptic estrogen receptor- $\alpha$ levels in prefrontal cortex in female rhesus monkeys and their correlation with cognitive performance. J Neurosci 30: 12770-12776.

Webster MJ, Knable MB, O'Grady J, Orthmann J, Weickert CS (2002). Regional specificity of brain glucocorticoid receptor mRNA alterations in subjects with schizophrenia and mood disorders. Mol Psychiatry 7: 985-994, 924.

Weiser MJ, Foradori CD, Handa RJ (2008). Estrogen receptor beta in the brain: from form to function. Brain Res Rev 57: 309-320.

Weiser MJ, Foradori CD, Handa RJ (2010). Estrogen receptor beta activation prevents glucocorticoid receptor-dependent effects of the central nucleus of the amygdala on behavior and neuroendocrine function. Brain Res 1336: 78-88.

Whitfield-Gabrieli S (2009). Region of Interest Extraction (REX) Toolbox. Boston, MA, USA. http://gablab.mit.edu/index.php/ research/95-gablab-site/gablab/people/swg.

Wingfield JC, Sapolsky RM (2003). Reproduction and resistance to stress: when and how. J Neuroendocrinol 15: 711-724.

Woolley CS (1998). Estrogen-mediated structural and functional synaptic plasticity in the female rat hippocampus. Horm Behav 34: 140-148.

Young EA, Midgley AR, Carlson NE, Brown MB (2000). Alteration in the hypothalamic- pituitary-ovarian axis in depressed women. Arch Gen Psychiatry 57: 1157-1162.

Young EA, Altemus M, Parkinson V, Shastry S (2001). Effects of estrogen antagonists and agonists on the ACTH response to restraint stress in female rats. Neuropsychopharmacology 25: 881-891.

Young EA, Korszun A (2002). The hypothalamic-pituitary-gonadal axis in mood disorders. Endocrinol Metab Clin North Am 31: 63-78.

Young EA, Ribeiro SC, Ye W (2007). Sex differences in ACTH pulsatility following metyrapone blockade in patients with major depression. Psychoneuroendocrinology 32: 503-507.

Zuloaga DG, Carbone DL, Quihuis A, Chong DL, Handa RJ (2012). Perinatal dexamethasone-induced alterations in apoptosis within the hippocampus and paraventricular nucleus of the hypothalamus are age- and sex-dependent. J Neurosci Res 90: 1403-1412.

Supplementary Information accompanies the paper on the Neuropsychopharmacology website (http://www.nature.com/npp) 